\title{
Limit-depth and DNR degrees
}

\author{
Philippe Moser ${ }^{\mathrm{a}, *}$, Frank Stephan ${ }^{\mathrm{b}}$ \\ a Department of Computer Science, National University of Ireland, Maynooth, Co Kildare, Ireland \\ b Department of Mathematics, The National University of Singapore, 10 Lower Kent Ridge Drive, S17, Singapore 119076, Republic of Singapore
}

\section{A R T I C L E I N F O}

\section{Article history:}

Received 13 September 2017

Received in revised form 13 February 2018

Accepted 15 February 2018

Available online 19 February 2018

Communicated by Marcin Pilipczuk

Keywords:

Theory of computation

Logical depth

Kolmogorov complexity

\begin{abstract}
A B S T R A C T
We introduce the notion of limit-depth, as a notion similar to Bennett depth, but well behaved on Turing degrees, as opposed to truth-table degrees for Bennett depth. We show limit-depth satisfies similar properties to Bennett depth, namely both recursive and sufficiently random sequences are not limit-deep, and limit-depth is preserved over Turing degrees. We show both the halting problem and Chaitin's omega are limit-deep. We show every limit-deep set has DNR wtt-degree, and some limit-cuppable set does not have a limit-deep wtt degree.
\end{abstract}

(C) 2018 Elsevier B.V. All rights reserved.

\section{Introduction}

Bennett [1] introduced logical depth as a way to measure computationally useful information as opposed to Kolmogorov random information. Bennett's notion has the following natural properties: Recursive sets are not Bennett deep (they only contain "trivial" patterns), Martin-Löf random sequences are not Bennett deep (they contain no patterns), and Bennett depth is preserved upwards by truthtable reductions, i.e. if set $S$ truth-table computes some Bennett deep set, then $S$ is also Bennett deep (this property is known as the slow growth law). The canonical example of Bennett deep vs non-deep set is given by the halting problem and Chaitin's omega. The halting problem and Chaitin's omega are Turing equivalent, yet the halting problem's information can be retrieved by polynomial time reductions, whereas to be useful, omega needs to be decompressed, and this decompression running time grows asymptotically larger than every recursive function. It is known that the halting problem is Bennett deep [1], and since omega is Martin-Löf random, it is not Bennett

\footnotetext{
* Corresponding author.

E-mail addresses: pmoser@cs.nuim.ie (P. Moser), fstephan@comp.nus.edu.sg (F. Stephan).
}

deep. Although these two sets are Turing-equivalent, this does not contradict the slow growth law, since they are not truth-table equivalent. Thus the appropriate reductions for Bennett depth are truth-table reductions, and Turing reductions are too general, as Turing degrees do not preserve Bennett depth.

However many results in recursion theory rely on more general reductions than truth table, and Turing reductions are ubiquitous in recursion theory. This motivates the present paper, in which we investigate what a depth notion à la Bennett would look like, if one chooses Turing reductions as the appropriate reductions (instead of truth-table). We propose a depth notion called limit-depth, which can be viewed as a version of Bennett depth at the level of Turing-reductions. For Bennett, a sequence is Bennett deep if for almost all its prefixes, the difference between time-bounded and standard Kolmogorov complexity is unbounded. In this paper we follow a similar idea, but consider the difference between standard Kolmogorov complexity and Kolmogorov complexity relative to the halting problem. We show that for every set, this difference of complexities is unbounded, therefore to obtain a meaningful notion, we consider sets for which this difference grows faster than some recursive order; we call such sets limit-deep. It turns out this notion satisfies similar natural properties to Bennett depth, namely: We show 
that sets that are random relative to the halting problem (a.k.a. 2-random sets), are not limit-deep. We show that limit-depth satisfies a slow growth law for weak truthtable reductions, i.e. limit-depth is closed upwards for wtt reductions. This implies that no recursive set is limit-deep. For Turing reductions, we show that the slow growth law holds for Turing degrees: If a set $A$ computes some limitdeep set, then the Turing degree of $A$ contains a limit-deep set.

We show that both the halting problem and Chaitin's omega are limit-deep. This is in stark contrast with Bennett depth (where omega is not Bennett deep), but is not surprising, since at the Turing reductions level, omega and the halting problem are equivalent, therefore it is expected for both to be limit-deep. This implies that limit-depth and Bennett depth are incomparable notions.

In [7] Moser and Stephan proved that every Bennett deep set is either high or DNR. Here we show limit-deep sets share a similar property, namely every limit-deep set has DNR wtt-degree.

It was shown in [7] that every Bennett deep set is high. We do not know if a direct analogue holds for limit-depth. However we show the analogy does not hold for limitcuppable sets, where a set is limit-cuppable if its join with the halting problem is Turing above the 2nd jump $\emptyset^{\prime \prime}$. We show that there exists a limit-cuppable set whose wtt degree does not contain any limit-deep set.

\section{Preliminaries}

We use standard computability/algorithmic randomness theory notations see $[2,8,9]$. We use $\leq^{+}$to denote less or equal up to a constant term. We fix a recursive 1-1 pairing function $\langle\cdot\rangle: \mathbb{N} \times \mathbb{N} \rightarrow \mathbb{N}$. We use sets and their characteristic sequences interchangeably. The join of two sets $A, B$ is the set $A \oplus B$ whose characteristic sequence is $A(0) B(0) A(1) B(1) \ldots$, that is, $(A \oplus B)(2 n)=A(n)$ and $(A \oplus B)(2 n+1)=B(n)$ for all $n$. An order function is an unbounded non-decreasing function from $\mathbb{N}$ to $\mathbb{N}$.

We consider standard Turing reductions $\leq_{T}$, and weak truth-table reductions $\leq w t t$ (where all queries are made in advance) and truth-table reductions $\leq_{t t}$ (total wtt reductions). Two sets $A, B$ are Turing equivalent $\left(A \equiv_{T} B\right)$ if $A \leq_{T} B$ and $B \leq_{T} A$. The Turing degree of a set $A$ is the set of sets Turing equivalent to $A$. Fix a standard enumeration of all oracle Turing machines $\varphi_{1}, \varphi_{2}, \ldots$. The jump $A^{\prime}$ of a set $A$ is the halting problem relative to $A$, i.e., $A^{\prime}=\left\{e: \varphi_{e}^{A}(e) \downarrow\right\}$. The halting problem is denoted $\emptyset^{\prime}$. A set $A$ is high (that is, has high Turing degree) if its halting problem is as powerful as the halting problem of the halting problem, i.e., $\emptyset^{\prime \prime} \leq_{T} A^{\prime}$. High sets are equivalent to sets that compute dominating functions (i.e., sets $A$ such that there is a function $f$ with $f \leq_{T} A$ such that for every recursive function $g$ and for almost every $n, f(n) \geq g(n)$ ), i.e., a set is high iff it computes a dominating function [9].

A total function $g$ is DNR if for every $e, g(e) \neq \varphi_{e}(e)$. A set is of DNR degree, if it computes a DNR function.

Fix a universal prefix free Turing machine $U$, i.e., such that no halting program of $U$ is a prefix of another halting program. The prefix-free Kolmogorov complexity of string $x$, denoted $K_{U}(x)$, is the length of the length- lexicographically first program $x^{*}$ such that $U$ on input $x^{*}$ outputs $x$. It can be shown that the value of $K_{U}(x)$ does not depend on the choice of $U$ up to an additive constant, therefore we drop $U$ from the notation and write $K(x)$. $K(x, y)$ is the length of a shortest program that outputs the pair $\langle x, y\rangle$, and $K(x \mid y)$ is the length of a shortest program such that $U$ outputs $x$ when given $y$ as an advice. If the universal TM $U$ is provided oracle access to the halting problem, we denote by $K^{\prime}$ and $C^{\prime}$ the corresponding complexities. $U^{-1}(x)$ denotes the set of programs $p$ such that $U(p)=x, U(p)[n] \downarrow=x$ means machine $U$ on input $p$ halts within $n$ steps of computation and outputs $x$, and $m(x)=\sum_{p \in U^{-1}(x)} 2^{-|p|}$ is the probability that $U$ outputs string $x$. It is well known that $\log m(x)={ }^{+}-K(x)$ (see e.g. [2]), the proof relies on the Kraft-Chaitin theorem which follows.

Theorem 1 (Kraft-Chaitin see e.g. [2]). Given an r.e. set of requests $\left\{\left\langle x_{i}, r_{i}\right\rangle: i \in \mathbb{N}\right\}$ (where $x_{i}$ are strings and $r_{i}$ are natural numbers) with $\sum 2^{-r_{i}} \leq 1$, there exists a prefix free machine $N$ with domain $\left\{p_{i}: i \in \overline{\mathbb{N}}\right\}$, such that for every $i, N\left(p_{i}\right)=x_{i}$ and $\left|p_{i}\right|=r_{i}$.

It is easy to check that the Kraft-Chaitin Theorem relativises to $\varnothing^{\prime}$.

Another consequence is the following symmetry of information result.

Theorem 2 (Symmetry of information see e.g. [2]). For every strings $x, y$, we have $K(x, y)={ }^{+} K(x)+K\left(y \mid x^{*}\right)$ where $x^{*}$ is a minimal $U$-program for $x$.

The proof of the symmetry of information theorem relativises to $\emptyset^{\prime}$.

A set $A$ is Martin-Löf random (MLR) if none of its prefixes are compressible by more than a constant term, i.e., $\forall n K(A\lceil n) \geq n-c$ for some constant $c$, where $A\lceil n$ denotes the first $n$ bits of the characteristic function of $A$. See [2,8] for more on $K$-complexity.

A set $A$ is order-complex [4] if its complexity is at least greater than some recursive order, i.e. there is a recursive order $h$ such that for every $n, K(A\lceil n) \geq h(n)$. It is known [4] that order-complex sets are exactly those sets whose wtt-degree is DNR.

Theorem 3 (Kjos-Hanssen, Merkle, and Stephan [4]). Set A is order-complex iff A wtt-computes a DNR function (iff $A$ ttcomputes a DNR function).

The following lemma is a relativised version of a similar result in [5].

Lemma 4. There is a constant $c$ such that for every string $x$ and every $p \in U^{-1}(x)$, we have $K^{\prime}(p) \leq|p|+\log (m(x))+$ $K^{\prime}(x)+c$.

Proof. Consider the following request set r.e. in $\emptyset^{\prime}$ : given $x^{*}$ the shortest $U^{\emptyset^{\prime}}$-program for $x$, simulate $U^{\emptyset^{\prime}}\left(x^{*}\right)$ to recover $x$. Compute $d=\lceil\log m(x)\rceil \leq 0$ using the oracle for $\emptyset^{\prime}$. 
Dovetail $U$ till the next $p$ is found with $U(p)=x$. Enumerate request $\langle p,|p|+d\rangle$. The weight of this request set is at most 1 because,

$\sum_{p \in U^{-1}(x)} 2^{-|p|-d} \leq 2^{-d} m(x) \leq 1$.

Let $N^{\emptyset^{\prime}}$ be the prefix free machine given by Theorem 1 . For every $p \in U^{-1}(x)$. We have $K_{N}(p) \leq|p|+\log m(x)$, thus $K^{\prime}(p) \leq^{+}|p|+\log m(x)+K^{\prime}(x)$.

\section{Limit-depth}

Intuitively a sequence is limit-deep if the difference in compression of its prefixes is greater than some recursive order.

Definition 5. Let $g(n) \leq n$ be an order. A set $S$ is $g$-limit-deep ${ }_{K}$ if for almost every $n \in \mathbb{N}, K\left(S\lceil n)-K^{\prime}(S \uparrow\right.$ $n) \geq g(n)$

A set is said limit-deep if it is $g$-limit-deep ${ }_{K}$ for some recursive order $g$. It is easy to see that for every two orders $f, g$ such that $\forall n \in \mathbb{N} f(n) \leq g(n)$, every g-limit-deep - $_{K}$ set is also $f$-limit-deep . $_{\text {. }}$

Bennett's original notion [1] considered $O(1)$ terms instead of order functions. Several authors have considered different order functions (see [6] for a summary) and as seen in [7] the choice has consequences on the computational power of the corresponding deep sets. Choosing $O(1)$ is not possible with limit-depth, because every sequence would qualify as limit-deep.

The following lemma follows from Solovay's work on $K^{\prime}$ [10] (see 10.2.6 in [2]); or the fact that $K^{\prime}(x)=$ $\lim \sup _{n} K(x \mid n)$. We include a proof using the KC theorem for completeness.

Lemma 6. For every $m \in \mathbb{N}$ and for almost every string $x$, $K(x)-K^{\prime}(x) \geq m$.

Proof. Let $m \in \mathbb{N}$. Consider the following r.e. in $\emptyset^{\prime} \mathrm{KC}$ set $L$, whose resulting prefix-free machine requires $c$ extra bits to be simulated by $U^{\emptyset^{\prime}}$ ( $c$ can be used in $L$ 's construction, by a standard application of the recursion theorem). $L=\{\langle x, K(x)-m-c\rangle: x>N\}$, where $N$ is least such that $\sum_{x>N} 2^{-K(x)} \leq 2^{-(m+c)}$. The weight of $L$ is less than 1 , and by choice of $c$, we have for every $x>N$, $K^{\prime}(x) \leq K(x)-(m+c)+c$.

Because the number $N$ in the previous proof is $\emptyset^{\prime}$ computable, the difference between $K$ and $K^{\prime}$ is greater than some $\emptyset^{\prime}$-recursive order. This explains why one needs to consider recursive orders to have a meaningful notion of limit-depth.

The following equivalent definition of limit-depth is useful to simplify some proofs.

Definition 7. Let $g(n) \leq n$ be an order. A set $S$ is $g$-limit-deep ${ }_{K}$ if for almost every $n \in \mathbb{N}$, and for every $p \in U^{-1}\left(A\lceil n), K^{\prime}(p) \leq|p|-g(n)\right.$.
Lemma 8. Definitions 5 and 7 are equivalent.

Proof. Suppose $S$ is $h$-limit-deep ${ }_{K}$ as per Definition 7, and let $n$ be large enough. Let $p \in U^{-1}(S\lceil n)$ be a shortest program for $S\left\lceil n\right.$, i.e. $|p|=K\left(S\lceil n)\right.$. Given a $U^{\emptyset^{\prime}}$-program for $p, U^{\emptyset^{\prime}}$ can recover $S\lceil n$, thus

$K^{\prime}\left(S\lceil n) \leq^{+} K^{\prime}(p) \leq|p|-h(n)=K(S\lceil n)-h(n)\right.$

i.e. $S$ is $(h-O(1))$-limit-deep ${ }_{K}$ as per Definition 5 .

Suppose $S$ is $h$-limit-deep - $_{K}$ as per Definition 5, and $n$ be large enough. Let $p \in U^{-1}(S\lceil n)$. By Lemma 4 we have $K^{\prime}(p) \leq^{+}|p|+\log m\left(S\lceil n)+K^{\prime}(S\lceil n)\right.$. Since $\log m(S \mid$ $n) \leq^{+}-K(S\lceil n)$, we have

$K^{\prime}(p) \leq^{+}|p|-K(S\lceil n))+K^{\prime}(S\lceil n) \leq|p|-h(n)$.

Bennett proved that MLR sets are not Bennett deep. The corresponding version for limit-depth is 2-randomness.

Theorem 9. Let $A$ be MLR relative to $\emptyset^{\prime}$. Then $A$ is not limitdeep.

Proof. Let $A$ be as above. Since $A$ is 2-random, one can find a sequence of natural numbers $\left\{k_{i}\right\}_{i}$ such that for every $i$ and every $n \geq k_{i}, K^{\prime}\left(A\left\lceil k_{i}\right)-k_{i} \leq K^{\prime}(A\lceil n)-n\right.$. Let $h$ be a recursive order function. Let $N=\{n: h(n-1)<h(n)\}$. $N$ is an infinite set such that for every $n \in N, K(n)<$ $h(n) / 2$ (a short program for $n$ says "find the smallest integer whose image under $h$ is $h(n)$ ", such a program can be encoded prefix free in $O(\log h(n))$ bits). Consider the prefix free machine $M$ which on input $q$ searches for the unique (if it exists) decomposition $q=q_{1} q_{2} q_{3}$ such that $U\left(q_{1}\right) \downarrow=r_{1},\left|q_{3}\right|=r_{1}$, and $U^{\emptyset^{\prime}}\left(q_{2}\right)\left[r_{1}\right] \downarrow$. If such a decomposition is found then $M$ outputs $U^{\emptyset^{\prime}}\left(q_{2}\right)\left[r_{1}\right] q_{3}$. Because $U$ is prefix free, so is $M$. Let $i \in \mathbb{N}$ and $k=k_{i}, p$ be a minimal $U^{\emptyset^{\prime}}$-program for $A \uparrow k$, let $r$ be the least natural number such that $U^{\emptyset^{\prime}}(p)[r]$ is stable, let $n \in N$ be the smallest with $n \geq r$, and let $n^{*}$ be a minimal $U$-program for $n$. Thus $M\left(n^{*} p A[k, k+n-1]\right)=A\lceil k+n$. Letting $m=k+n$ we have

$$
\begin{aligned}
K(A\lceil m) & \leq{ }^{+} K_{M}\left(A\lceil m) \leq\left|n^{*}\right|+|p|+n\right. \\
& =K(n)+K^{\prime}(A\lceil k)+m-k \\
& \leq K(n)+K^{\prime}\left(A\lceil m) \leq h(n) / 2+K^{\prime}(A\lceil m) .\right.
\end{aligned}
$$

Since $h$ is arbitrary, $A$ is not limit-deep.

Bennett's slow growth law (SGL) [1] states that non Bennett deep sequences cannot compute deep ones quickly (i.e. by a truth table reduction). The following result shows that for limit-depth, a similar result holds for wtt reductions.

Lemma 10. Let $h$ be a recursive order, and $A \leq_{w t t} B$ be two sets. If $A$ is $h$-limit-deep ${ }_{K}$ then $B$ is $h^{\prime}$-limit-deep $_{K}$ for some recursive order $h^{\prime}$. Furthermore given indices for the weak truth-table reduction and for $h$, one can effectively compute an index for $h^{\prime}$. 
Proof. Let $A, B$ be as above, let $\varphi$ denote the wtt reduction, and let $f$ be a recursive order such that for every $n, \varphi\left(B\lceil n)=A\left\lceil f(n)\right.\right.$. Fix $n \in \mathbb{N}$ and let $p \in U^{-1}(B\lceil n)$. Since $(\varphi \circ U)(p)=A \uparrow f(n)$, there is a program $q$ of constant size (independent of $n$ ) such that $U(q p)=A\lceil f(n)$, i.e. $q p \in U^{-1}\left(A\lceil f(n))\right.$. Since $A$ is $h$-limit-deep , $_{K}$, we have

$K^{\prime}(p) \leq{ }^{+} K^{\prime}(q p) \leq|q p|-h(f(n))={ }^{+}|p|-(h \circ f)(n)$

i.e. $B$ is $(h \circ f)$-limit-deep ${ }_{K}$.

\section{Corollary 11. No recursive set is limit-deep.}

Proof. Let $A$ be recursive and $R$ be 2-random. We have $A \leq{ }_{w t t} R$, thus $\mathrm{A}$ is not limit-deep.

Note that Theorem 10 does not hold for $\leq_{T}$, since there are sets in the Turing-degree of $\Omega$ which are not ordercomplex, e.g. use $\Omega$ to compute a dominating function $h$ and construct a sequence with long runs of 0 s (length of $n$-th run is at least $h(n))$ i.e. $A$ is not order complex, and each runs of zeroes are separated by a 1 (resp. 11) depending on whether the next bit of $\Omega$ to code is a 0 (resp. a 1 ). However the slow-growth law holds for $\leq_{T}$ on degrees.

Theorem 12. Let $A \leq_{T} B$ be two sets, where $A$ is limit-deep. Then there exists a limit-deep set $C \equiv_{T} B$.

Proof. Let $A, B$ be as above, and let $h$ be a recursive order witnessing $A$ being limit-deep (wlog we can assume $h$ is $o(n))$. Define $E=\{n: \log h(n)>\log h(n-1)\}=\left\{n_{0}<n_{1}<\right.$ $\left.n_{2}<\ldots\right\}$ and $g(n)=|E \cap[0, n]| . g$ is a recursive order with $g(n) \leq \log h(n)$ for every $n$. Consider the recursive order $l(n)=h(n-g(n))-2 \log h(n)$. The idea is to define $C$ to be $A$, except a few number of far separated bits (measured by $g$ ) used to code $B$. More precisely let

$C(n)= \begin{cases}B(i) & \text { if } n=n_{i} \\ A(n-g(n)) & \text { otherwise }\end{cases}$

Thus $C \uparrow n$ codes $g(n)$ bits of $B$ and $n-g(n)$ bits of $A$, thus $K^{\prime}\left(C\lceil n) \leq^{+} K^{\prime}\left(A\lceil n-g(n))+2 g(n)\right.\right.$, and $K\left(C\lceil n) \geq^{+}\right.$ $K(A\lceil n-g(n))$. We have

$K\left(C\lceil n)-K^{\prime}(C\lceil n) \geq h(n-g(n))-2 g(n)=l(n)\right.$

Clearly $A \equiv_{T} B$, which ends the proof.

No MLR sequence is Bennett deep. This contrasts with limit-depth, where some MLR sequences can be limit-deep.

Theorem 13. $\Omega$ is limit-deep.

Proof. Since $\Omega$ is recursive in $\emptyset^{\prime}$, for every $n$ we have $K^{\prime}\left(\Omega\lceil n) \leq^{+} K^{\prime}(n) \leq 2 \log n\right.$. Since $\Omega$ is MLR, for every $n$ we have $K\left(\Omega\lceil n) \geq n-O(1)\right.$, thus $\Omega$ is $h$-limit-deep ler $_{\text {lim }}$ for $h(n)=n / 2$.

Corollary 14. The halting problem is limit-deep.
Proof. Follows from Lemma 10 and $\emptyset^{\prime} \leq{ }_{w t t} \Omega$.

The halting problem is both Bennett deep and limitdeep. On $\Omega$ the two notions differ. The following result shows the two notions are incomparable.

Theorem 15. Bennett depth and limit-depth are incomparable notions.

Proof. By Theorem 13, $\Omega$ is limit-deep but not Bennett deep. For the converse it is know that there exists Schnorr random ultracompressible sequences [5], i.e. sets $A$ such that for every recursive order $g, K(A\lceil n) \leq K(n)+g(n)$ for all but finitely many $n$, but for every recursive time bound $t, K^{t}(A\lceil n) \geq n-g(n)+O(1)$, for all but finitely many $n$. Thus $A$ is Bennett deep. Fix a recursive order $g$. We have $K\left(A\lceil n)-K^{\prime}\left(A\lceil n) \leq K(n)-K^{\prime}(n)+g(n) / 2\right.\right.$. Since the difference $K(n)-K^{\prime}(n)$ can be made less than every recursive order infinitely often (see proof of Theorem 9 ), there are infinitely many $n$ with $K\left(A\lceil n)-K^{\prime}(A\lceil n) \leq g(n) / 2\right.$, i.e. $A$ is not $g$-limit-deep. Since $g$ is arbitrary, $A$ is not limitdeep.

Since order-complex sets have DNR wtt-degrees, it follows that limit-deep sets have DNR wtt-degrees.

Theorem 16. Every limit-deep set has DNR wtt-degree.

Proof. Notice that every limit-deep sequence is ordercomplex and apply Theorem 3.

It was shown in [7] that every Bennett deep set is high. A natural analogue for limit-depth would be generalised high 1 sets (denoted GH1) i.e., sets $A$ such that $A^{\prime} \equiv_{T}\left(A \oplus \emptyset^{\prime}\right)^{\prime}$. Although we do not know the answer for GH1 sets, we show that the analogy with Bennett deep sets fails to hold in the setting of limit-depth, if one considers limit-cuppable sets.

Definition 17. A set $A$ is limit-cuppable if $A \oplus \emptyset^{\prime} \geq_{T} \emptyset^{\prime \prime}$.

Theorem 18. There exists a limit-cuppable set whose wtt degree does not contain any limit-deep set.

Proof. We use the following jump theorem: If $C>0$ and $D \geq_{T} \emptyset^{\prime} \oplus C$ then there exists a 1 -generic set $A$ such that $A^{\prime} \equiv_{T} A \oplus C \equiv_{T} D$ (see e.g. [2]). Letting $D=\emptyset^{\prime \prime}, C=\emptyset^{\prime}, A$ is a 1-generic limit-cuppable set. Because generic sets contain arbitrary long sequences of $0 \mathrm{~s}, A$ is not order-complex, thus its wtt-degree does not contain a DNR function by Theorem 3, thus it does not contain a limit-deep set by Theorem 16.

\section{Final remark}

We conclude this paper with a series of questions. As noted in Theorem 18, it would be interesting to see if an analogue of "high implies Bennett deep" holds for limitdepth. Also it is known [3,7] that low (r.e.) Bennett deep 
sets exist. What about limit depth; are all low (r.e.) sets not limit-deep? Can Theorem 9 be improved to weak 2 random sets?

We thank the referees for useful comments and some further open questions.

\section{References}

[1] Charles H. Bennett, Logical depth and physical complexity, in: The Universal Turing Machine, A Half-Century Survey, 1988, pp. 227-257.

[2] Rodney G. Downey, Denis R. Hirschfeldt, Algorithmic Randomness and Complexity, Springer, 2010.

[3] Rod Downey, Michael McInerney, Keng Meng Ng, Lowness and logical depth, Theor. Comput. Sci. 702 (2017) 23-33.
[4] Björn Kjos-Hanssen, Wolfgang Merkle, Frank Stephan, Kolmogorov complexity and the recursion theorem, Trans. Am. Math. Soc. 363 (2011) 5465-5480.

[5] David W. Juedes, James I. Lathrop, Jack H. Lutz, Computational depth and reducibility, Theor. Comput. Sci. 132 (1994) 37-70.

[6] Philippe Moser, On the polynomial depth of various sets of random strings, Theor. Comput. Sci. 477 (2013) 96-108.

[7] Philippe Moser, Frank Stephan, Depth, highness and DNR degrees, Discret. Math. Theor. Comput. Sci. 19 (4) (2017).

[8] André Nies, Computability and Randomness, Oxford University Press, 2009.

[9] Piergiorgio Odifreddi, Classical Recursion Theory: The Theory of Functions and Sets of Natural Numbers, vol. 1, Elsevier, 1989.

[10] R.M. Solovay, Draft of a paper (or series of papers) on Chaitin's work. Unpublished notes. May 1975. 